\title{
A determination of the source of Jovian hectometric radiation via occultation by Ganymede
}

\author{
W. S. Kurth, ${ }^{1}$ S. J. Bolton, ${ }^{2}$ D. A. Gurnett,${ }^{1}$ and S. Levin ${ }^{2}$
}

\begin{abstract}
During the first Galileo flyby of Ganymede, Jovian radio emissions in the frequency range of $700 \mathrm{kHz}$ to $5.6 \mathrm{MHz}$ were completely occulted by the satellite. We take advantage of this serendipitous observation to determine the location of the radio emission source relative to Jupiter. The evidence suggests that the apparent source is along an $L \gtrsim 7$ magnetic field line near the central meridian longitude $\left(\sim 160^{\circ}\right)$ of the spacecraft and at 1 to 3 Jovian radii above the northern hemisphere of Jupiter. These results are consistent with a source located along either the Ganymede or Europa flux tube.
\end{abstract}

\section{Introduction}

The first Galileo flyby of Ganymede revealed that this satellite has an internal magnetic field [Kivelson et al., 1996] and a magnetosphere [Gurnett et al., 1996]. In addition, Jovian radio emissions up to $5.6 \mathrm{MHz}$ were completely occulted by Ganymede for periods lasting up to about 24 minutes. We use the precise positions of Galileo, Ganymede, and Jupiter at the times of these occultations to locate the position of the source relative to Jupiter as a function of frequency.

The radio emissions of interest here lie between about $700 \mathrm{kHz}$ and the $5.6 \mathrm{MHz}$ upper frequency limit of the instrument. Emissions in this frequency range are usually referred to as hectometric radiation since the wavelengths are on the order of 100 meters. Extensive observations of these emissions were carried out by the Voyager Planetary Radio Astronomy investigation [c.f. Alexander et al., 1981; Ladreiter and Leblanc, 1991] and the Ulysses Unified Radio and Plasma Wave (URAP) investigation [Stone et al., 1992]. The URAP instrument has an upper frequency cutoff of $940 \mathrm{kHz}$, hence, it could observe only the lower frequency portion of these emissions. However, the URAP instrument has the capability to perform two-dimensional direction-finding measurements on radio emissions and was used to determine the location of hectometric emissions. Results from these studies yielded locations above the Jovian auroral zone on field lines ranging from $L \approx 6$ [Reiner et al., 1993a,b] to $\mathrm{L} \approx 11$ [Ladreiter et al., 1994]. The current model for the generation of hectometric radiation is that it is generated in the high latitude, low altitude region near Jupiter via the cyclotron maser instability [c.f. Ladreiter and Leblanc, 1991].

The Galileo plasma wave instrument utilizes an electric dipole antenna mounted on the end of the 10.6$\mathrm{m}$ magnetometer boom with a length of approximately

\footnotetext{
${ }^{1}$ Department of Physics and Astronomy, University of Iowa, Iowa City.

${ }^{2}$ Jet Propulsion Laboratory, Pasadena.

Copyright 1997 by the American Geophysical Union.
}

Paper number $97 \mathrm{GL} 00988$.

0094-8534/97/97GL-00988\$05.00
$6.6 \mathrm{~m}$ tip-to-tip. Search coil magnetometers covering the frequency range up to $160 \mathrm{kHz}$ are also used, mounted on the high gain antenna feed, but due to their limited frequency range are irrelevant to the current topic. The plasma wave receiver includes 158 spectral channels operated as a sweep frequency receiver covering the range from $5.62 \mathrm{~Hz}$ to $5.62 \mathrm{MHz}$, which accumulates a complete spectrum every 18.67 seconds. For a more detailed description of the instrument, see Gurnett et al. [1992].

Kaiser and Alexander [1976] were the first to use occultations by a moon to carry out radio directionfinding measurements. They used occultations of the Earth by the moon to determine the source position of the Earth's auroral kilometric radiation with RAE-2 and used the duration of time for the radio signal to disappear or reappear as an indication of the source size. At a given frequency the Ganymede ingress and egress occur over time periods on the order of a few 18.67-s sample periods, hence, we can apply basically the same technique as Kaiser and Alexander. Since Galileo has several frequency channels across the hectometric frequency range, we can also map the distribution of the emission source as a function of frequency.

\section{The Occultation}

Figure 1 is a frequency-time spectrogram showing the intensity of waves as a function of frequency and time using the color bar on the right to relate wave intensity to various colors. Blue represents the lowest intensity and red the highest intensity. For context, we have included the data down to $300 \mathrm{~Hz}$ so that the primary interaction between Ganymede's magnetosphere and Jupiter's magnetosphere can be seen [Gurnett et al., 1996]. Closest approach to Ganymede occurred at about 0630 spacecraft event time (SCET).

Of primary interest is the complete occultation of the hectometric radiation between about 0550 and 0624 SCET. Both the ingress and the egress appear to be extremely sharp, on the order of only a few 18.67 -s instrument sweeps. Such a complete and sharply-defined occultation can only be explained by a single small source. The occultation is, however, frequency dependent in that the higher frequencies are occulted first and longest. This implies that the source location is frequency dependent.

The method of direction-finding by occultation is quite simple. As illustrated in Figure 2, we show Jupiter as the small body at the center of the figure and the limb of Ganymede at four different times by the four larger circles. The smaller pair of these represent the limb of Ganymede at 0559:30 and 0602:40 during which time the $1.0-\mathrm{MHz}$ emission was occulted. The larger pair of circles represent the limb of Ganymede at 0620:20 and 0621:00 SCET when the emission reappeared. The source, then, must be one of the two regions of inter- 


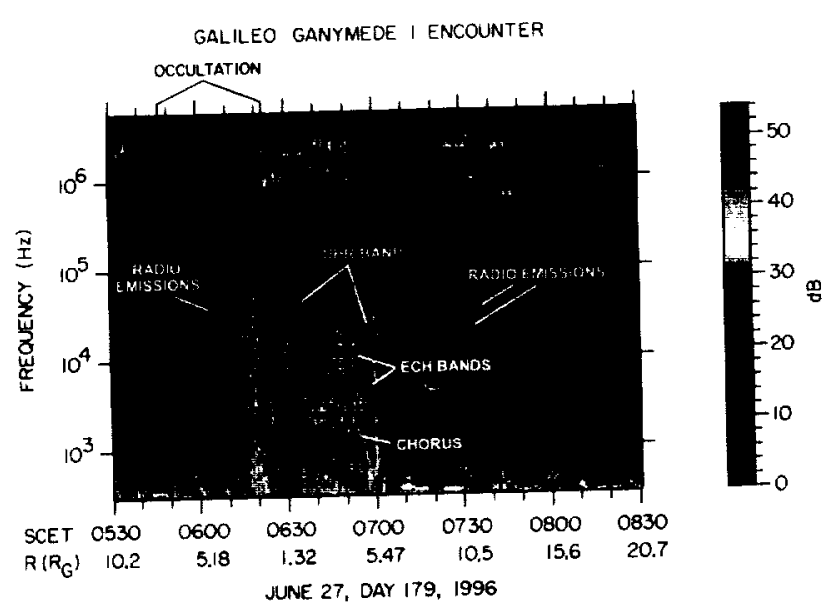

Figure 1. A frequency-time spectrogram showing the dramatic plasma and radio wave signature of Ganymede's magnetosphere and the sharp, total occultation of the Jovian hectometric radiation.

section of the four Ganymede limbs. Since it is highly unlikely that the two small sources were occulted simultaneously, one of them represents the real source. We plot both but based on earlier studies, we assume the source situated over Jupiter's north auroral zone is the actual source. During the occultation, the magnetic latitude of Galileo ranged from $6^{\circ}$ to more than $8^{\circ}$ north, so it is less likely that southern hemisphere emissions are being observed at this time.

Also in Figure 2 we show the results for 3.6 and 4.8 $\mathrm{MHz}$. The three pairs of regions show the source sizes observed, ranging from regions of order $0.1 R_{J}$ at the lower frequencies to elongated regions of order $1 R_{J}$ at higher frequencies. The determination of the earliest and latest times for the ingresses and egresses is confused to some extent by temporal variations in the intensity of the radio emission. The regions plotted in Figure 2 probably reflect both the actual source size plus any errors associated with choosing the times. In the limit in which the errors dominate, the regions are more accurately thought of as error bars on the direction determination. In the opposite limit in which there is minimal timing error, the region would truly be the extent of the source.

We do not consider either the ephemeris data or the size of Ganymede (the radius of $2634 \mathrm{~km}$ is thought to be within $10 \mathrm{~km}$ of its true value [IAU/IAG/Cospar, 1996]), to be significant contributors to the errors. It is clear from Figure 2, however, that the limb of Ganymede is very large at egress (just before closest approach) and, as shown by the $4.8 \mathrm{MHz}$ region, it is moving very rapidly. Using the length of this region and the egress duration of $90 \mathrm{~s}$, the angular rate of motion of the limb as viewed by Galileo is about $0.6 \mathrm{mrad} / \mathrm{s}$. Hence, timing errors will be most important for high frequencies at egress. Therefore, we believe that the elongated nature of the $4.8-\mathrm{MHz}$ result is primarily due to timing inaccuracies and is not indicative of the true source extent.

One other source of error we have not considered quantitatively is the effect of refraction near the source, in the Io torus, or in Ganymede's ionosphere. Menietti and Reiner [1996] have recently shown that refraction can be substantial for propagation out of the meridian

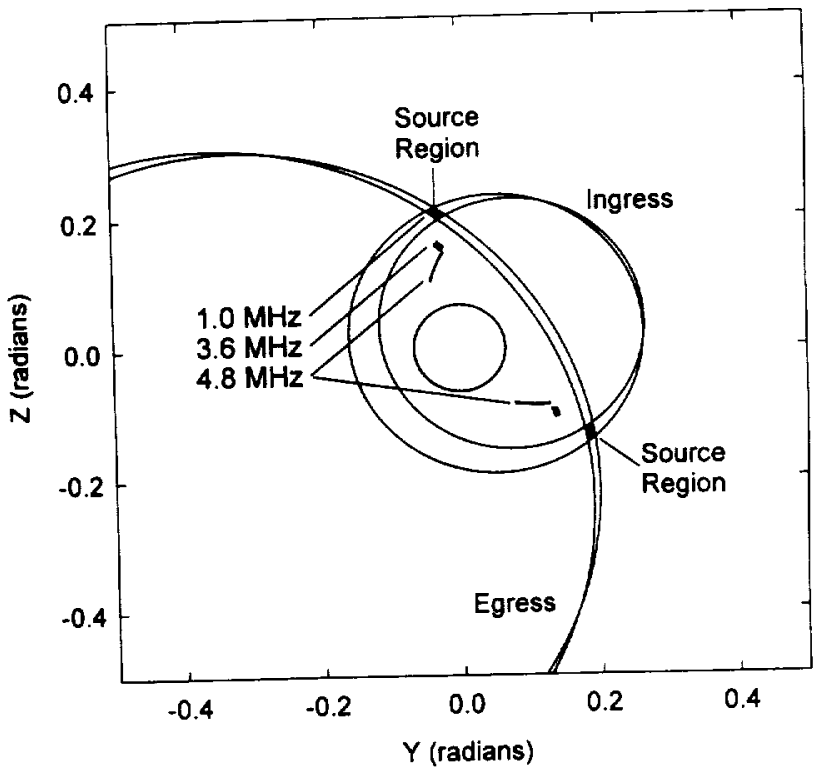

Figure 2. The determination of source region extent based on the earliest and latest ingress and egress times at $1.0,3.6$, and $4.8 \mathrm{MHz}$.

plane. However, our results suggest propagation in or close to the meridian plane, hence, the primary refraction would be in the $\mathrm{Z}$ direction. We discuss possible refraction effects below.

In Figure 3 we plot the midpoints of the source regions for several frequencies from $710 \mathrm{kHz}$ to $5.62 \mathrm{MHz}$. The locus of solutions is along a line extending radially outward from Jupiter with the highest frequency solutions closest to Jupiter, consistent with emission along a field line via the cyclotron maser instability just above

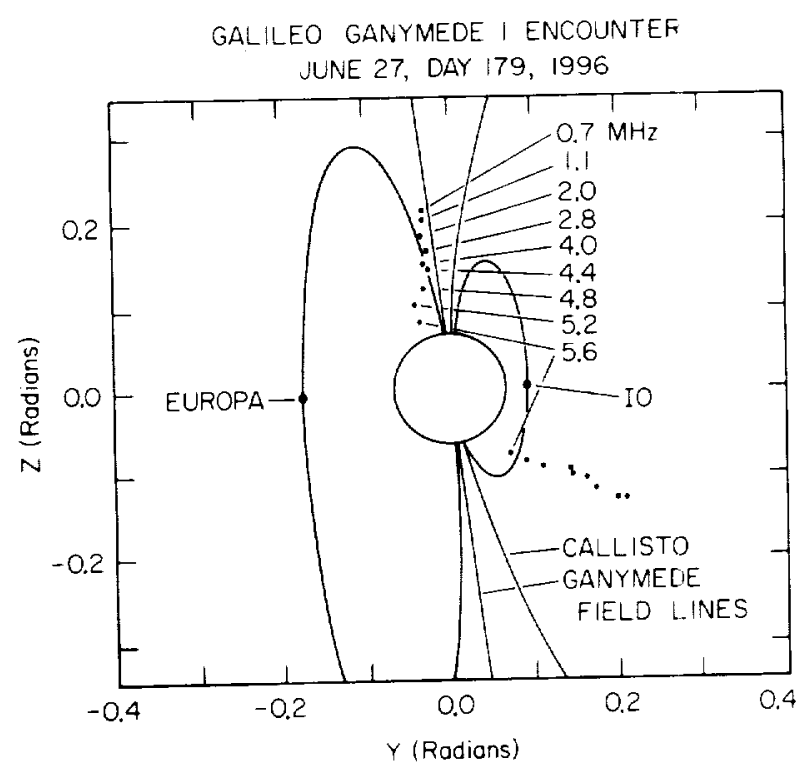

Figure 3. Based on analyses similar to that shown in Fig. 2, a series of source locations can be derived as a function of frequency. Note that the sources align reasonably well with both the field lines that thread Ganymede and Europa. Field lines through Io and Callisto are also shown. 
the electron cyclotron frequency. It is likely that the skewing of the midpoints of the high-frequency source locations to the east in Figure 3 is a result of the timing errors discussed above.

\section{Discussion}

Also in Figure 3 we have included some specific field lines based on the 06 model without a current sheet [Connerney, 1993]. We make the reasonable assumption that the nearly linear locus of source positions should lie along the source field line. The field line which appears as nearly a straight line is that which threads Ganymede near a System III longitude ( $\left.\lambda_{\text {III }}\right)$ of $158^{\circ}$ and $L \approx 15$ at the time of the occultation. The other field lines are Europa's at $\lambda_{\text {III }} \approx 164^{\circ}$ and $L \approx 9.3$, Io's at $357^{\circ}$ longitude and $L \approx 5.9$, and Callisto's at $129^{\circ}$ and $L \approx 26.5$. At this time Galileo is at $\lambda_{I I I} \approx 158^{\circ}$ and $15.2 \mathrm{R}_{\mathrm{J}}$. The alignment of the source directions with the Ganymede and Europa field lines is fairly good, suggesting that the source must be very close to the one or the other of these two satellite's flux tubes at the time of the occultation.

In Figure 4 we plot the directions determined in Figure 3 as viewed from a vantage point rotated 90 degrees, or about 70 degrees System III longitude. We have also plotted 158 degree field lines with $L$ values of 6,8 , and 15. The primary point of this construction is to illustrate that field lines of $L<7$ are not consistent with the apparent position of the observed lowest frequency source locations and one must rely on larger field lines. However, we cannot assess an upper limit to $\mathrm{L}$. We have also plotted contours of constant electron cyclotron frequency $f_{\text {ce }}$ in Figure 4 to show where the source directions intersect the illustrated field lines. For the higher frequency directions ( $\gtrsim 4.8 \mathrm{MHz}$ ), the solutions intersect a family of field lines with $\mathrm{L} \gtrsim 7$ at about the appropriate $f_{\mathrm{ce}}$ contour. For the middle frequency solutions, however, the solutions do not intersect the appropriate contour at all. We do not have a good explanation for why these solutions intersect field lines where the

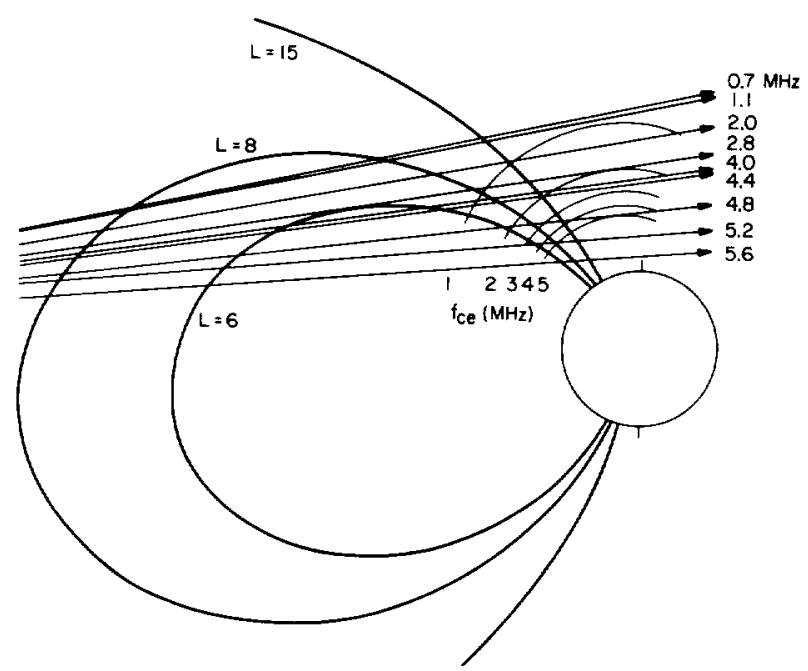

Figure 4. A side view of the results shown in Figure 3 illustrating the source altitude above Jupiter. Note that field lines with $L \gtrsim 7$ must be considered in order for the sources at all frequencies to fall on a single field
line. cyclotron frequency is too low to support emission at that frequency, unless the observed emissions are harmonics. The 1.1- and $0.7-\mathrm{MHz}$ solutions approach the $1-\mathrm{MHz}$ contour, but only at very high latitudes.

It is possible that refraction plays a role, especially at the lower frequencies. Since the sources we discuss lie northward of high density regions near Jupiter and propagate northward of the Io torus, we would expect refraction away from these regions. The result of refraction from these sources should be to place the actual source even farther north than the apparent direction. This would exacerbate the inconsistency with the electron cyclotron harmonic contours. Even though the measured plasma frequencies $(\sim 100 \mathrm{kHz})$ in Ganymede's immediate vicinity are quite low compared to the hectometric radiation frequencies, some refraction may occur. The result of this refraction is different if the density increases steadily with decreasing altitude than if there are high density layers such as in Earth's ionosphere. In the former case, refraction should be away from Ganymede, again consistent with placing actual source locations farther north than the apparent direction. Layering, however, could have the opposite effect. No layering was observed in the in situ density measurements from either of the first two Ganymede encounters, and the second encounter closest approach was only $260 \mathrm{~km}$ above the surface.

Finally, given that the field lines near $7<\mathrm{L}<15$ are the source, one can see from Figure 4 that the emission cone opening angle is of order $40^{\circ}$. This is consistent with previous studies (c.f. Ladreiter et al., 1994].

The very sharp and total occultation observed during the Ganymede flyby leads to the conclusion that at a given frequency there is one very small source. Further, the apparent alignment with a single or small range of field lines near the central meridian longitude of Galileo certainly raises the question as to what would make a particular field line so significant. We suggest two possible answers to this question. First, a single, small source could be explained if the emission is beamed outward in the central meridian plane. Assuming emission in a hollow cone around the source field line as is often assumed for the cyclotron maser instability, the only way we can envision such a restriction would be for the hollow cone to be oriented such that only the southern-most extension of the cone is oriented toward Galileo and that emission in other directions around the cone are beamed to higher latitudes than Galileo. This would seem to be highly unlikely since the condition would have to exist over the entire range of frequencies observed. Second, one would have to consider either the Europa or Ganymede flux tubes as possibly connected to the source region. One could imagine that the dramatic interaction between Ganymede and the Jovian magnetosphere might drive currents similar in nature to those driven by Io, albeit at a somewhat lower level. Hence, it is possible or even expected that such an interaction might drive radio emissions near the Jovian high latitude region. We know of no previous studies indicating any Ganymede control over hectometric emissions, but this is something that should be pursued. The results of the first Europa encounter are not yet analyzed, hence, it is too early to speculate on the possible interactions with that moon.

Another unusual feature of the Ganymede occultation is that only a single source was observed. Ladre- 
iter et al. [1994] suggest that there are probably multiple sources extended over a range of longitudes and Reiner et al. [1993a] suggest that the source could be distributed over 25 degrees in longitude. Why only one is seen at this time is not understood. Further, from the accumulated set of hectometric radiation observations obtained by Galileo to date, it is clear that hectometric radiation is observed even when the spacecraft and Ganymede or Europa are on opposite sides of the planet. Certainly propagation from a source region such as that determined here across the Jovian pole to Galileo would be highly unlikely, unless the emission is generated in the ordinary mode as well as the extraordinary mode. To date, polarization studies of the hectometric radiation consistently conclude the emission is generated primarily in the extraordinary mode with some weaker ordinary mode emission. We cannot rule out other source locations for the hectometric radiation at other times based on this single observation.

\section{Conclusions}

We have used the occultation of Jovian hectometric radiation by Ganymede to do a precise direction-finding determination of the radiation source as a function of frequency. The source appears to lie along an $L \gtrsim 7$ field line near the central meridian plane of the spacecraft of about $160 \lambda_{\text {III }}$ at a distance of $1-3 R_{J}$ from Jupiter over the northern auroral zone for frequencies between 5.6 and $0.7 \mathrm{MHz}$, respectively. The apparent source directions are not consistent with emission at the electron cyclotron frequency for frequencies between about 2 and $4.5 \mathrm{MHz}$, however, suggesting that there may be some refraction affecting the results. The source size at a given frequency is small, of order $0.1 R_{J}$. The results are consistent with one of two possible conclusions. First, the source could be on a single or highly localized set of magnetic field lines threading Ganymede or Europa. The very strong interaction between the magnetospheres of Jupiter and Ganymede would certainly seem consistent with processes that might produce radio emissions. Second, the emission may simply propagate in the meridian plane and only the source at Galileo's central meridian longitude is visible to Galileo at the time of the occultation.

Ladreiter et al. [1994] report central meridian longitudes for the sources they studied in the range of $40^{\circ}$ to $130^{\circ}$ and Reiner et al. [1993a] found a range of $30^{\circ}$ to $115^{\circ}$. The $158^{\circ}$ reported here is seemingly inconsistent with these results. However, Lecacheux et al. [1992] showed that the peaks in hectometric radiation occurrence probability in $\lambda_{\text {III }}$ are a function of the latitude of the observer. This implies there is a strong latitudinal beaming and that the latitude of the observer is important in setting the $\lambda_{\text {III }}$ range for optimal hectometric visibility. Since during this time period Galileo was orbiting with an inclination that favored southern latitudes, the $\lambda_{1 I I}$ range required to place it in a latitude-limited beam would be different from most previous studies (e.g. Voyagers 1 and 2 and Ulysses inbound) that were based on trajectories biased to the north.
We should also point out that the observations presented herein were confined to a single 30 -minute time period. At other times there may be emissions from locations other than the Ganymede or Europa field lines.

Acknowledgment. The research at the University of Iowa is supported by NASA through contract 958779 with the Jet Propulsion Laboratory.

\section{References}

Alexander, J. K., T. D. Carr, J. R. Thieman, J. J. Schauble, and A. C. Riddle, Synoptic observations of Jupiter's radio emissions: Average statistical properties observed by Voyager, J. Geophys. Res., 86, 8529-8545, 1981.

Connerney, J. E. P., Magnetic fields of the outer planets, J. Geophys. Res., 98, 18,659-18,679, 1993.

Gurnett, D. A. et al.. The Galileo plasma wave investigation, Space Sci. Rev., 60, 341-355, 1992.

Gurnett, D. A., W. S. Kurth, A. Roux, S. J. Bolton, and C. F. Kennel, Ganymede's magnetosphere: Galileo plasma wave observations, Nature, 384, 535-537, 1996.

IAU/IAG/Cospar, Report of the IAU/IAG/Cospar working group on cartographic coordinates and rotational elements of the planets and satellites, Celestial Mechanics and Dynamical Astronomy 63, pp. 127-148, 1996.

Kaiser, M. L. and J. K. Alexander, Source location measurements of terrestrial kilometric radiation obtaincd from lunar orbit, J. Geophys. Res., 3, 37-40, 1976.

Kivelson, M. G. et al., Discovery of Ganymede's magnetic field by the Galileo spacecraft, Nature, 384, 537-541, 1996.

Ladreiter, H. P., and Y. Leblane, The Jovian hectometric: radiation: An overview after the Voyager mission, Ann. Geophysicae 9, 784-796, 1991.

Ladreiter, H. P., P. Zarka, and A. Lecacheux, Direction finding study of Jovian hectometric and broadband kilometric radio emissions: Evidence for their auroral origin, Planct. Space Sci., \7, 919-931, 1994.

Lecachenx, A. et al., In ecliptic observations of Jovian radio emissions by Ulysses: Comparison with Voyager results, Geophys. Res. Lett., 19, 1307-1310, 1992.

Menietti, J. D., and M. J. Reiner, Modeling of Jovian hectometric radiation source locations: Ulysses observations, J. Geophys. Res., 101, 27,045-27,052, 1996.

Reincr, M. J., J. Fainlerg, and R. G. Stonc, Source characteristics and locations of hectometric radio emissions from the northern Jovian hemisphere, Geophys. Res. Lett., 20, 321-324, 1993a.

Reiner, M. J., J. Fuinberg, and R. G. Stone, Sourec characteristics and locations of Jovian hectometric radio emissions, J. Geophys. Res., 98, 18,767-18,777, 1993l.

Stone, R. G. ct al., The unified radio and plasma wave investigation, Astron. and Astrophys. Supp. Ser., 9\%, 291-316, 1992.

D. Gurnett and W. Kurth, Department of Physics and Astronomy, University of Iowa, Iowa City, IA 52242. (e-mail: donald-gurnett@iniowa.edu; williamkurth@uiowa.edu)

S. Bolton and S. Levin, Jet Propulsion Laboratory, 4800 Oak Grove. Drive, Pasadena, CA 91109. (e-mail: sbolton@gllsve.jpl.nasa.gov; steve@beta.jpl.nasa.gov)

(Received January 22, 1997; revised March 6, 1997; accepted March 12, 1997.) 